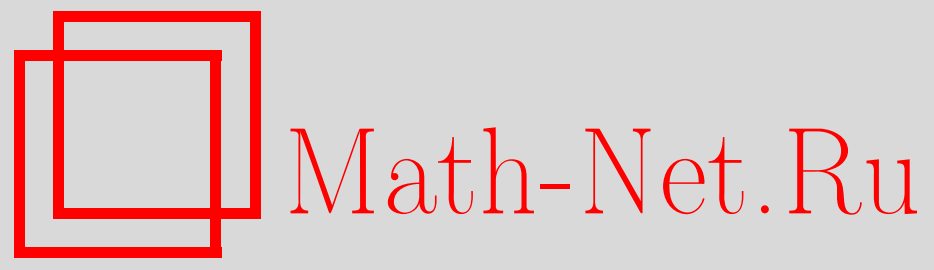

Е. И. Бережной, А. А. Перфильев, Точная теорема экстраполяции для операторов, Функи. анализ $и$ его прил., 2000, том 34, выпуск 3, 66-68

DOI: https://doi.org/10.4213/faa313

Использование Общероссийского математического портала MathNet.Ru подразумевает, что вы прочитали и согласны с пользовательским соглашением

http://www . mathnet.ru/rus/agreement

Параметры загрузки:

IP : 54.198 .55 .26

26 апреля 2023 г., 09:13:40

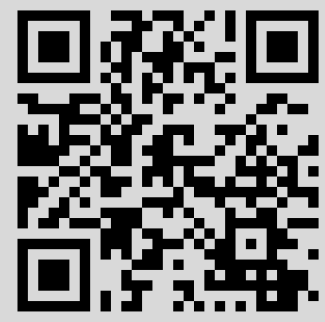




\title{
Точная теорема экстраполяции для операторов
}

\author{
(C) 2000. Е. И. БЕРЕЖНОЙ, А. А. ПЕРФИЛЬЕВ
}

Первая теорема экстраполяции была предложена Яно [1] в 1951 г. Теорема Яно утверждает, что линейный оператор, нормы которого допускают оценку $\left\|T \mid L^{p} \rightarrow L^{p}\right\| \leqslant c /(p-1)^{s}$, где $p \in(1,2], c$ не зависит от $p$ и $s>0$ - фиксированное число, ограниченно действует из $L^{1}(\log L)^{s}$ в $L^{1}$. С помощью этой теоремы Яно удалось показать ограниченность оператора максимальной функции Харди-Литлвуда и оператора сопряженной функции из $L^{1}(\log L)^{1}$ в $L^{1}$. Независимо близкие к теореме Яно результаты были получены в $[2,3]$.

Позднее Керман [4] заменил выражение $\delta /(p-1)^{s}$ в теореме Яно на выражение $\left(p^{2} /(p-1)\right) b\left(e^{p^{2} /(p-1)}\right)$, где $b(t)$ удовлетворяет некоторым ограничениям, и написал интегральное неравенство, которому в этом случае удовлетворяет оператор T. Большая работа Яверта и Мильмана [5] посвящена рассмотрению теоремы экстраполяции с точки зрения общих идей теории интерполяции линейных операторов ( $K$ - и $J$-методов действительной интерполяции).

В настоящей работе предложен новый подход к доказательству теорем экстраполяции для сублинейных операторов. В результате найдено короткое доказательство точной теоремы экстраполяции, в условиях которой удалось заменить $L^{1}$ на произвольное банахово пространство, а функцию $1 /(p-1)^{s}-$ на произвольную возрастающую функцию.

Пусть $(U, \Sigma, \mu)$ - пространство с $\sigma$-конечной мерой и $\chi(D)$ - характеристическая функция множества $D$. Для $f: U \rightarrow \mathbb{R}$ через $\lambda(f, \gamma)$ обозначим функцию распределения функции $f: \lambda(f, \gamma)=\mu(\{t \in U:|f(t)|>\gamma\})$, а через $f^{*}$ ее перестановку в невозрастающем порядке. Банахово пространство $X$ измеримых функций называется симметричным [6], если из включения $f \in X$, измеримости функции $g$ и из выполнения при всех $\gamma \in \mathbb{R}_{+}$неравенства $\lambda(g, \gamma) \leqslant \lambda(f, \gamma)$ следует, что $g \in X$ и $\|g|X\|\leqslant\| f| X\|$. Пусть $W$ - множество непрерывных вогнутых функций $\psi: \mathbb{R}_{+} \rightarrow \mathbb{R}_{+}, \psi(0)=0$. Пространство Лоренца $\Lambda(\psi)$ (пространство Марцинкевича $M(\psi))$ состоит из тех $f$, для которых конечна норма

$$
\begin{gathered}
\|f \mid \Lambda(\psi)\|=\int_{0}^{\infty} f^{*}(s) d \psi(s)=\int_{0}^{\infty} \psi(\lambda(f, s)) d s \\
\left(\|f \mid M(\psi)\|=\sup _{0<t<\infty} \frac{1}{\psi(t)} \int_{0}^{t} f^{*}(s) d s=\sup _{D \subseteq U}\left\{\frac{1}{\psi(\mu(D))} \int_{D}|f(s)| d s\right\}\right) .
\end{gathered}
$$

Пространства Лоренца и Марцинкевича являются, наряду с $L^{p}$, классическими примерами симметричных пространств. Если $\psi(t)=t^{\theta}$ с $0<\theta<1$, то пространство Лоренца (Марцинкевича) в этом случае обозначается через $\Lambda^{\theta}\left(M^{\theta}\right)$. Теория симметричных пространств функций подробно изложена в $[6,7]$.

Теорема 1. Пусть $Y$ - некоторое банахово пространство, $T$ - определенный на множестве $Q$ конечнозначных функций линейный оператор $T: Q \rightarrow Y$ и $\varphi:[1, \infty) \rightarrow \mathbb{R}_{+}$- возрастающая функция, $\varphi(1)=1$. Для $а \in$ $(0, \infty)$ определим функцию

$$
\psi_{\varphi}(a)=\inf _{t \geqslant 1}\left\{a^{1-1 /(t+1)} \varphi(t)\right\} .
$$


Предположим, что при всех $p \in(1,2]$ для любого $D \subseteq U$ выполнено неравенство

$$
\|T \chi(D) \mid Y\| \leqslant c(\mu(D))^{1 / p} \varphi(1 /(p-1))
$$

с константой с, не зависящей от $D$ и р.

Тогда для любой функиии $f \in Q$ верно неравенство

$$
\left\|T f|Y\|\leqslant 2 c\| f| \Lambda\left(\psi_{\varphi}\right)\right\| .
$$

ДоКАЗАТЕЛЬСТво. Для каждого фиксированного $t \geqslant 1$ функция $\theta(a)=$ $a^{1-1 /(t+1)} \varphi(t)$ является вогнутой на $\mathbb{R}_{+}$. Поэтому функция $\psi_{\varphi}(a)$ как нижняя грань вогнутых функций тоже будет вогнутой. Непосредственно из определения функции $\psi_{\varphi}(a)$ следует, что $\lim _{a \rightarrow 0} \psi_{\varphi}(a)=0$. Поэтому $\psi_{\varphi} \in W$ и, следовательно, определено пространство $\Lambda\left(\psi_{\varphi}\right)$.

Из леммы 5.2 монографии [6, с. 151] следует, что для любой функции $\psi \in W$ справедливы соотношения

$$
\begin{aligned}
\sup \left\{\frac{\|T \chi(D) \mid Y\|}{\|\chi(D) \mid \Lambda(\psi)\|}: D \subseteq U\right\} & \leqslant \sup \left\{\frac{\|T f \mid Y\|}{\|f \mid \Lambda(\psi)\|}: f \in Q\right\} \\
& \leqslant 2 \sup \left\{\frac{\|T \chi(D) \mid Y\|}{\|\chi(D) \mid \Lambda(\psi)\|}: D \subseteq U\right\} .
\end{aligned}
$$

Зафиксируем $D \subseteq U$. Так как неравенство (2) справедливо для всех $p \in(1,2]$, то, полагая $p=1+1 / t$ и минимизируя по $t$, придем к неравенству $\|T \chi(D) \mid Y\| \leqslant$ $c \psi_{\varphi}(\mu(D))=c\left\|\chi(D) \mid \Lambda\left(\psi_{\varphi}\right)\right\|$. Применяя (4), получим (3).

Теорема доказана.

Учитывая определение функции $\psi_{\varphi}(a)$ и соотношения (4), теорему 1 можно переформулировать в следующем виде.

Теорема 1'. Пусть $Y$ - некоторое банахово пространство, $T$-определенный на множестве $Q$ линейный оператор, $T: Q \rightarrow Y, u \varphi:[1, \infty) \rightarrow \mathbb{R}_{+}-$ возрастаюшая функиия, $\varphi(1)=1$. Для того чтобь при всех $p \in(1,2]$ выполнялось неравенство

$$
\left\|T f\left|Y\left\|\leqslant c_{1} \varphi(1 /(p-1))\right\| f\right| \Lambda^{1 / p}\right\|
$$

c некоторой константой $c_{1}$, не зависящей от $p$ u $f \in Q$, необходимо и достаточно, чтобь выполнялось неравенство (3), где функиия $\psi_{\varphi}($ a) определена равенством (1).

Справедливость двойственной теоремы к теореме 1 следует из двойственности пространств Лоренца и Марцинкевича [6].

Теорема 2. Пусть $Y$ - некоторое банахово пространство, $T$ - линейньй оператор, $T: Y \rightarrow S(U, \Sigma, \mu)$, где $S(U, \Sigma, \mu)$ - пространство измеримьх на $(U, \Sigma, \mu)$ функиий, и $\varphi:[1, \infty) \rightarrow \mathbb{R}_{+}-$возрастающая функиия, $\varphi(1)=1$. Для того чтобы при всех $p \in(1,2]$ выполнялось неравенство

$$
\left\|T \mid Y \rightarrow M^{1 / p}\right\| \leqslant c \varphi(1 /(p-1))
$$

с константой с, не зависящей от р, необходимо и достаточно, чтобь выполнялось неравенство

$$
\left\|T \mid Y \rightarrow M\left(\psi_{\varphi}\right)\right\|<\infty
$$

где функция $\psi_{\varphi}($ (а) определена равенством (1). 
ПримеР 1. Пусть функция $\varphi$ при всех $t \in(1, \infty)$ удовлетворяет неравенству

$$
\frac{1}{c} t \varphi^{\prime}(t) \leqslant \varphi(t) \leqslant c t \varphi^{\prime}(t) \text {. }
$$

Положим

$$
\psi(a)=a \begin{cases}\varphi(\ln (1 / a)) / \varphi(2) & \text { при } a \in\left(0, e^{-2}\right), \\ 1 & \text { при } a \in\left[e^{-2}, 1\right], \\ \sqrt{a} \varphi(1) & \text { при } a \in(1, \infty) .\end{cases}
$$

Следует заметить, что функция $\psi(a)$ не всегда вогнута.

$\mathrm{C}$ помощью прямых вычислений получим, что при всех $a>0$ справедливо соотношение

$$
\frac{1}{c_{1}} \psi_{\varphi}(a) \leqslant \psi(a) \leqslant c_{1} \psi_{\varphi}(a)
$$

с константой $c_{1}$, не зависящей от $a$.

Отметим, что условию (5) удовлетворяют, например, функции $\varphi(t)=t^{\alpha}$, $\varphi(t)=t^{\alpha}(1+\ln t)^{\beta_{1}}$ и $\varphi(t)=t^{\alpha}(1+\ln t)^{\beta_{1}}(\ln (e+\ln t))^{\beta_{2}}$ при $\alpha, \beta_{1}, \beta_{2} \in \mathbb{R}_{+}$.

Используя пример 1 , из теоремы 1 получим следующее утверждение.

Tеорема 3. Пусть $\mu(U)<\infty, Y$ - некоторое банахово пространство u $T$ - определенный на множестве $Q$ линейный оператор, $T: Q \rightarrow Y$, для которого при любом $D \subseteq U$ вьполнено неравенство (2). Пусть $\varphi:[1, \infty) \rightarrow \mathbb{R}_{+}$ возрастающая функиия, $\varphi(1)=1$, и для нее выполнено неравенство (5).

Тогда выполняется неравенство (3), где функиия $\psi_{\varphi}$ при мальх а определена равенством (6).

Если в теореме 3 положить $Y=L^{1}, \varphi(t)=t^{\alpha}, \alpha>0$, то получается теорема экстраполяции из [5], обобщающая результат Яно. В этом случае $\Lambda\left(\psi_{\varphi}\right)$ совпадает с $L^{1}\left(\log L^{1}\right)^{\alpha}$.

ЗАмечАниЕ. Теоремы $1,1^{\prime}$ и 3 этой работы справедливы не только для линейных, но и для сублинейных операторов. Теорема 2 также будет справедливой и для сублинейных операторов, если $Y$ - симметричное пространство. Напомним, что определенный на $L^{1, \operatorname{loc}}(U)$ оператор $T$ называется сублинейным, если для абсолютной константы $c_{0}$ на области его определения почти всюду выполнены неравенства

$$
|T \lambda f| \leqslant c_{0}|\lambda||T f|, \quad\left|T\left(\sum f_{k}\right)\right| \leqslant c_{0} \sum\left|T f_{k}\right| .
$$

\section{ЛИТЕРАТУРА}

1. Yano S. J. Mat. Soc. Japan., 3, 296-305 (1951). 2. Юдович В. И. ДАН СССР, 138, № 6, 805-808 (1961). 3. Симоненко И. Б. Матем. сб., 63, № 4, 536-553 (1964). 4. Kerman R. A. Studia Math., 76, 183-195 (1983). 5. Jawerth B., Milman M. Mem. Amer. Math. Soc., 89, No. 440, 1-82 (1991). 6. Крейн С. Г., Петунин Ю. И., Семенов Е. М. Интерполяция линейных операторов. Наука, М., 1978. 7. Lindenstrauss J., Tzafriri L. Classical Banach spaces. Vol. 1, 2. Springer-Verlag, Berlin-Heidelberg-New York, 1977, 1979. 